\title{
Correction to: Chromosome instability induced by mutations in TAD anchors leads to tumors
}

\author{
Andrey N. Luchnik ${ }^{1}$ [
}

Published online: 3 December 2021

(c) Shenzhen University School of Medicine; Fondazione Istituto FIRC di Oncologia Molecolare 2021

\section{Correction to: Genome Instability \& Disease https://doi.org/10.1007/s42764-021-00050-1}

Due to a misunderstanding during proof correction, some references were cited in false positions in the article.

In particular the sections "Inlaid genome" and "SMC complexes" were affected. The original article has been corrected.

Springer Nature apologizes to the readers of the journal for not detecting the mistakes during the production process.

The original article can be found online at https://doi.org/10.1007/ s42764-021-00050-1.

Andrey N. Luchnik

luchnik.andrey@yandex.ru

1 Koltzov Institute of Developmental Biology, Russian Academy of Sciences, Moscow, Russia 